\title{
Taxi Service
}

National Cancer Institute

\section{Source}

National Cancer Institute. Taxi Service. NCI Thesaurus. Code C141289.

A transportation service that uses hired cars and drivers. 